\title{
The sarcopenic risk factor of low skeletal muscle mass is associated with inadequate plasma vitamin $C$ concentrations and obesity.
}

\section{Abstract}

Sarcopenia, characterised by loss of skeletal muscle mass and strength with age, is a significant risk factor for loss of mobility and independence. The combination of low muscle mass and high fat mass in sarcopenic obesity is associated with particularly poor outcomes. Micronutrient deficiencies can occur alongside obesity despite total energy surplus, and older individuals may be at greater risk of deficiency. Research suggests vitamin $\mathrm{C}$ is important for musculoskeletal health, but the relationship with obesity is underexplored.

This study aimed to investigate associations of plasma vitamin $\mathrm{C}$ with obesity status and explore the relationship with the sarcopenic risk factor, low skeletal muscle mass.

EPIC-Norfolk cohort study data were analysed. Bioelectrical impedance analysis-estimated fat free mass (FFM; a proxy for skeletal muscle mass) was adjusted for BMI to give a scaled variable, $\mathrm{FFM}_{\mathrm{BMI}}$. A 'low muscle mass' category was defined as individuals with the lowest $10 \% \mathrm{FFM}_{\mathrm{BMI}}$, representing those at high risk of sarcopenia. Plasma vitamin $\mathrm{C}$ (ascorbic acid) concentrations were categorised as inadequate $(<50 \mathrm{micromol} / \mathrm{L})$ or adequate $(\geq 50 \mathrm{micromol} / \mathrm{L})$, and obesity status as non-obese $\left(<30 \mathrm{~kg} / \mathrm{m}^{2}\right)$ or obese $\left(\geq 30 \mathrm{~kg} / \mathrm{m}^{2}\right)$.

Individuals were grouped according to vitamin $C$ and obesity status: 1 , non-obese and adequate vitamin $C$; 2 , non-obese and inadequate vitamin $\mathrm{C}$; 3 , obese and adequate vitamin $\mathrm{C}$; and 4 , obese and inadequate vitamin $\mathrm{C}$. Using logistic regression, the odds ratio (OR) of each vitamin/obesity status group was calculated in relation to membership of the 'low muscle mass' category. Analyses were sex-stratified and adjusted for age, smoking status, physical activity, social class, menopausal and HRT status in women, statin use, and corticosteroid use.

Data were analysed for 5903 men (mean 62.9 years, SD 9.0) and 7416 women (mean 61.5 years, SD 9.0). Prevalence of vitamin C inadequacy was higher in obese vs non-obese individuals (men $45.8 \%$ vs $33.0 \%$; and women $26.0 \%$ vs $15.3 \%$ ). The odds of 'low muscle mass' were higher in all vitamin/obesity status groups vs group 1, but the greatest odds were seen for group 4 (combined obesity and inadequate vitamin C) in men (OR 16.5, 95\% CI: 12.6-21.6; p<0.001) and women (OR 30.2, 95\% CI: 23.0-39.8; p < 0.001).

In this cohort of older individuals higher prevalence of vitamin $\mathrm{C}$ inadequacy is associated with obese individuals. Of importance to musculoskeletal health and our understanding of sarcopenia is the observation that while vitamin $\mathrm{C}$ inadequacy and obesity are each independently important, their coexistence is a particularly strong predictor of sarcopenic risk.

\section{Conflict of Interest}

There is no conflict of interest. 